\title{
Stroke management during the COVID-19 outbreak: challenges and results of a hub-center in Lombardy, Italy
}

\author{
Francesco Asteggiano ${ }^{1}$ • Ignazio Divenuto ${ }^{1}$ - Daniele Ajello ${ }^{1}$. Nicolò Gennaro ${ }^{2,3}$ - Orazio Santonocito ${ }^{2,3}$. \\ Simona Marcheselli ${ }^{4}$. Luca Balzarini ${ }^{3} \cdot$ Nunzio Paolo Nuzzi $^{1} \cdot$ Letterio Salvatore Politi $^{1,2}$ (D)
}

Received: 3 September 2020 / Accepted: 1 December 2020 / Published online: 7 January 2021

(C) The Author(s), under exclusive licence to Springer-Verlag GmbH, DE part of Springer Nature 2021

\begin{abstract}
Purpose We describe the reorganization carried out during the COVID-19 outbreak at one of the stroke centers in Italy and report on the clinical features and procedural variables of stroke patients in need of endovascular treatment.

Methods From 1 March to 10 May 2020, we retrospectively analyzed data from stroke patients in need of urgent neurointerventional treatment. Clinical presentation, demographics, and clinical history were collected along with procedural variables (door-to-needle time, needle-to-mTiCi time). Each patient underwent a nasal swab (polymerase chain reaction test), clinical screening, and chest CT scan to assess the risk of SARS-CoV-2 infection. Technical success, procedural safety (including staff SARS-CoV-2 infection), and clinical outcome at discharge were retrieved. A comparison was made with the same patient population treated between 1 March and 10 May 2019 to highlight possible differences in the characteristics or outcomes of the patients.

Results One hundred thirty-six ischemic stroke patients were admitted to our facility from 1 March to 10 May 2020. Of these, 12 patients (9\%) were classified as "high risk" for SARS-CoV-2 infection. Radiological suspicion of COVID-19 was confirmed in all cases by pharyngeal swab. Five SARS-CoV-2 patients (42\%) needed endovascular therapy. None of the staff members tested positive for IgG against SARS-CoV-2. Compared to the same period in 2019, an increase in the mean interval from the first symptoms to hospital arrival was observed $(p<0.05)$.

Conclusion Endovascular treatment of stroke presented several challenges during the COVID-19 outbreak. Within the hospital, special pathways can be used to maintain both procedural safety and procedural times.
\end{abstract}

Keywords Stroke $\cdot$ COVID-19 $\cdot$ Thrombectomy $\cdot$ Italy

\section{Background}

Italy has been the first nation in Europe to deal with the coronavirus disease 2019 (COVID-19) outbreak in late February

NNP and PLS provided equal contribution and share senior authorship

Letterio Salvatore Politi

letterio.politi@hunimed.eu

1 Neuroradiology Department, Humanitas Clinical and Research Center - IRCCS, via Manzoni 56, 20089 Rozzano, Italy

2 Department of Biomedical Sciences, Humanitas University, Pieve Emanuele, Italy

3 Radiology Department, Humanitas Clinical and Research Center IRCCS, Rozzano, Italy

4 Stroke Unit, Neurology Department, Humanitas Clinical and Research Center - IRCCS, Rozzano, Italy
2020, being the first case of SARS-COV-2 infection reported in Milan on February 18, 2020 [1]. Three months after, on 20 May 2020, Lombardy registered a total of 85,775 cases with 15,662 deaths, whereas the metropolitan city of Milan accounted for 22,372 cases, being the general death rate nearly doubled compared to the same period in 2019 [2]. In the early days of the epidemic outbreak, the Regional Healthcare System established a strict standardization for the management of time-sensitive diseases to optimize the resources during the COVID-19 outbreak. As a consequence, our institution was designed as one of the two hub-centers to treat cerebral ischemic stroke in the metropolitan city of Milan (3,250,000 inhabitants). In a scenario where the only available data regarding the relation between COVID-19 and stroke events were available as pre-prints only [3, 4], we aimed at providing safe, time-effective pathways for all patients being accepted to the neuroradiology department, including acute 
stroke patients [5]. Here, we report the clinical characteristics and outcome of stroke patients requiring urgent endovascular treatment for acute cerebral ischemia in this particular period.

\section{Methods}

This study has been reviewed and approved by the institutional review ethical committee with respect to scientific content and compliance with applicable research and human subject regulations.

We retrospectively reviewed the clinical data of stroke patients in need of urgent neurointerventional treatment who were admitted to the emergency department of our facility from 1 March to 10 May 2020. We report demographics data and neurological presentation including NIHSS, side, and site of occlusion. All patients underwent pre-procedural chest CT to assess the risk of SARS-Cov-2 infection. According to typical clinical symptoms (i.e., fever, cough, anosmia, dysgeusia, diarrhea) and typical radiological findings (i.e., peripheral ground-glass pulmonary consolidations, hilar adenopathies, pleural effusion), patients were stratified in "low-risk" and "high-risk" for COVID-19, being considered "high risk" all patients presenting at least one of the abovementioned symptoms or radiological findings [5]. Then, we report time intervals between the onset of neurological impairment and hospital admission (onset-to-door time), hospital admission and groin puncture (door-to-groin time), and groin puncture and $\mathrm{mTiCi}$ (needle-to-mTiCi time). Procedural variables including technical success and complications were then analyzed; technical success was defined according to mTiCi scale (mTiCi 2b-3) [6]. In the case of positive SARSCov-2 patients, we report the procedural safety defined as SARS-Cov-2 to staff by analyzing specific IgG-anti-SARSCov-2 at the end of May 2020.

\section{Results}

\section{Reorganization plan}

In early March 2020, multidisciplinary meetings were organized between the directors of the emergency department, radiology and neuroradiology departments, intensive care unit, stroke unit, and clinical facilities to adopt new guidelines for the management of COVID-19 patients, in particular with a view to establishing safe and time-effective pathways. At the entrance of the hospital, all health workers were equipped with surgical masks. In addition, health professionals involved in the stroke pathway received specific training on safety measures in the e-learning platform and specific simulation sessions in small groups. For SARS-CoV-2 patients, separate routes and workflows were adapted to achieve complete isolation from non-SARS-CoV-2 patients. The departments of radiology and neuroradiology were divided into two separate areas, with special areas and special equipment (X-ray, CT scan) for infected or highly suspicious COVID-19 patients. Due to the specific needs of patients with acute stroke, a special pathway for stroke code was chosen (Fig. 1). All stroke patients who came to the emergency room received a surgical mask and underwent a throat swab (polymerase chain reaction test) in the triage and were considered potentially infected with COVID-19. Patients presenting with neurological symptoms, and that were referred by the territorial emergency team as "stroke code," received immediate neurological evaluation at admission. With the exception of cases with already diagnosed COVID-19, the imaging protocol, including non-improved head CT scans, perfusion CT, and angiography, was supplemented with a chest $\mathrm{CT}$ scan to identify typical findings of interstitial pneumonia. In the event of a radiological suspicion of COVID-19, dedicated personnel immediately decontaminated the CT room. If the diagnosis of stroke with indication for endovascular intervention was confirmed, the stroke endovascular team was alerted. If the risk for COVID-19 was considered low, thrombectomy was performed as usual in the neuroangiography suite equipped with a biplane angiograph (Siemens Artis Zee, Forchheim, Germany). After the procedure, isolated stroke unit beds were dedicated for stroke patients until the result of the SARSCoV-2 swab previously performed in the emergency department. When the patent was deemed "high risk" for COVID-19 (presenting at least one of the following symptoms, fever, cough, anosmia, dysgeusia, or diarrhea, or radiological findings, peripheral ground-glass pulmonary consolidations, hilar adenopathies, or pleural effusion), thrombectomy was performed by neurointerventional radiologists who were adequately equipped with personal protective equipment, including FFp2/FFp3 masks, anti-x masks for eye protection, double sterile gloves, non-sterile waterproof clothing, sterile surgical suits, and disposable shoe covers [7]. After the procedure, a special team provided immediate decontamination of the neuroangio suite. Those SARS-CoV-2 positive patients who did not need intensive care were collocated in a COVID department under the strict supervision of the stroke unit neurology team. Those whose conditions were critical were collocated in a dedicated COVID-19 intensive care unit.

\section{Stroke population and COVID-19 infection}

In the period from 1 March to 10 May 2020, 833 patients with confirmed COVID-19 infection were observed in our facility. During the same period, 136 ischemic strokes were admitted to the emergency department, with $12(9 \%)$ patients with nasal swabs (polymerase chain reaction test) confirmed to be SARS-CoV-2 patients. The mean age of stroke patients who tested positive for SARS-CoV-2 was $66.1( \pm 10.8 \mathrm{SD})$, while 


\section{Suspected SARS-CoV-19 infection}

$\downarrow$

- Incoming at triage

- Mask the Patient

- Clinical examination with neurological impairment and suspicion of stroke

- Nasal and pharyngeal swab

- ECG and hematological screening

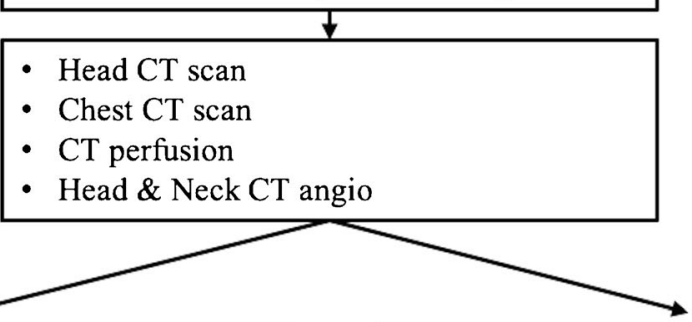

High Risk (likely Sars-Cov-2 positive)

Low Risk (likely Sars-Cov-2 negative)

Fever or evidence of SARS-CoV-19

Pneumonia, LVO occlusion

- Perform thrombectomy as high risk (maximum level of protective PPE)

- In-hospital stay in Covid-19 dedicated division.
No clinical suspicion, no evidence of SARS-CoV-

19 Pneumonia, LVO occlusion

- Perform thrombectomy as low risk (normal protective clothes)

- In-hospital stay in Covid-19 free division (when swab excludes infection).

Fig. 1 Reorganization plan for suspicious SARS-CoV-2 patients in an Italian stroke center. LVO, large vessel occlusion

the mean age of non-COVID-19 stroke patients was 75.4 ( \pm 8.8 SD). Of the SARS-CoV-2 patients, $5(42 \%)$ showed a large vessel occlusion (two on the right, three on the left) requiring endovascular recanalization, including 4 patients with M1 occlusion and 1 patient with tandem occlusion (Tables 1 and 2).

NIHSS at presentation was in all cases $>10$ (range 10-16). Of the 124 non-SARS-CoV-2 patients, only 28 (22\%) were suitable for endovascular recanalization. In 14 out of 33 endovascular procedures (42\%), tissue plasminogen activators (0.9 mg / kg alteplase) were administered immediately after diagnosis. All procedures were performed under conscious sedation, except one where a massive alteplase-related oral bleeding required intubation to protect the airways.

Table 1 Patients treated in 2019 and 2020 with LVO

\begin{tabular}{llll}
\hline & COVID- & COVID- & Patients 2019 \\
& $19+2020$ & 19 & \\
\hline$n$ & 12 & 124 & 65 \\
LVO & $5(42 \%)$ & $28(22 \%)$ & $7(10.7 \%)$ \\
\hline
\end{tabular}

Table 2 Clinical characteristics of patients treated during the COVID19 outbreak with LVO

\begin{tabular}{lll}
\hline LVO procedures & & \\
\hline & Covid-19+ & Covid-19- \\
\hline$n$ & 5 & 28 \\
Age & 66.1 & 75.2 \\
Male sex & 5 & 10 \\
NIHSS $>$ 10 & & \\
Before thrombectomy & 5 & 20 \\
After thrombectomy & 1 & 3 \\
MRS 0-1 & 4 & 25 \\
MRS 0-1 (3 months) & 4 & 20 \\
Hypertension & 3 & 15 \\
Dyslipidemia & 1 & 7 \\
Diabetes & 2 & 1 \\
Occlusion site & & \\
M1 & 2 & 12 \\
M2 & 0 & 8 \\
Basilar & 1 & 3 \\
Tandem & 2 & 5 \\
\hline
\end{tabular}




\section{Observations in stroke response}

As a 24/7 hub-center, we counted on four interventional neuroradiologist-team, which did not undergo downsizing during the emergency. We recorded a significant increase in neuroangiographic activity from 7 thrombectomies performed between 1 March and 10 May 2019 to 33 procedures in the same period of 2020. Of the latter, 5 patients $(12 \%)$ were SARS-Cov-2 positive (Table 2). In 2019, the mean interval between the onset of symptoms and the start of intervention was $217 \mathrm{~min}$, while it increased to $365 \mathrm{~min}$ during the COVID-19 outbreak. In 2019, the break-in time was $105 \mathrm{~min}$ and door-to-door time $112 \mathrm{~min}$, while the break-in time and door-to-door time were $248 \min (p<0.05)$ and $117 \min (p=0.3)$, respectively. No significant modification of the needle-to-mTiCi was observed, with a median revascularization time of $15 \mathrm{~min}$. Technical success was achieved in 25 patients (75\%). Demographics and clinical data of patients are shown in Tables 1 and 2. No physician or staff involved in neurointerventional stroke procedures tested positive for SARS-CoV-2 at the end of May 2020.

\section{Discussion}

The COVID-19 outbreak has severely impaired clinical activity and forced a reorganization of hospital networks for the management of neurological emergencies such as the stroke $[5,8]$. Concern about stroke patients was also highlighted by the possible effects of SARS-CoV-2 infection on the pathogenesis of cerebral ischemia in the brain $[9,10]$. In the period of March-April 2020, 9\% of ischemic strokes treated in our department were SARS-Cov-2 patients. Despite procedural technical success, which did not change significantly compared to the same period in 2019, we observed a significant increase in the time between hospital admissions (248 vs. $105 \mathrm{~min}, p<0.05)$. We can identify three main reasons for the dilatation of the pre-hospital interval time. First, people's anxiety may have discouraged patients from going to hospital. Secondly, the available ambulances were overloaded due to the simultaneous COVID-19 emergency, which also required time-consuming decontamination processes. Third, patients were asked to take longer distances from their homes to the specified hospitals, as only two centers were identified in the entire metropolitan area. Our analysis confirms the data from the Comprehensive Barcelona Stroke Center [11] which did not observe significant changes in the time intervals of inpatient treatment of stroke patients and in the time of procedure. All stroke patients were first examined for COVID-19 by chest $\mathrm{CT}$. With this analysis, we were able to quickly determine which patient is to be considered "highly suspicious" for COVID-19 infection and a possible source of contamination in the hospital. In this way, the risks to staff and other patients were minimized without significantly lengthening the door-togroin time, and the sanification was carried out only when necessary. The results we present confirm that this choice was effective both in terms of time (groin to $\mathrm{mTiCi}$ ) and in terms of protection (no infection between medical staff). Our course of action is in line with the model proposed by the SNIS Society [12] and Smith et al. [13] with the exception of breast CT scans for pneumonia associated with COVID-19.

There is emerging evidence in literature that the prevalence of stroke in COVID-19 infected patients is as high as $15 \%$ [14]. In our experience, a total of 833 COVID-19 patients were admitted in our hospital. Of the COVID-19 positive patients, only 12 presented an acute stroke. On the other hand, of all the 136 acute stroke patients admitted, only $9 \%$ were positive for the SARS-CoV-2 infection. Our data suggest a trend of a higher prevalence of large vessel occlusions in younger patients with COVID-19 infection that however is not statistically significant in our study [15]. We have seen no hospital major strokes in COVID-19 infected patients, not even in patients with serious terminal conditions in intensive care.

Despite the low number of thrombectomies we performed in SARS-CoV-2 patients, we did not report any intraprocedural complications, including technical difficulties or anesthesiological problems. Furthermore, also the adopted measures of self-protection did not affect the performance of the operators in terms of technical success and recanalization time.

In conclusion, despite the rapid reorganization of the clinical workflow during the COVID-19 outbreak, preand intraprocedural stroke management can be successfully maintained. SARS-Cov-2 patients admitted after a stroke showed a trend to be younger and to present a large vascular occlusion requiring endovascular therapy. Future studies with larger cohorts are needed to determine the impact of SARS-CoV-2 on the clinical outcome of patients with endovascular recanalization in acute cerebral ischaemia.

Funding No funding was received for this study.

\section{Compliance with ethical standards}

Conflict of interest The authors declare that they have no conflict of interest.

Ethical approval All procedures performed in the studies involving human participants were in accordance with the ethical standards of the institutional and/or national research committee and with the 1964 Helsinki Declaration and its later amendments or comparable ethical standards.

Informed consent Informed consent was obtained from all individual participants included in the study. 


\section{References}

1. WHO Director-General's opening remarks at the media briefing on COVID-19 - 11 March 2020. https://www.who.int/dg/speeches/ detail/who-director-general-s-opening-remarks-at-the-mediabriefing-on-covid-19\%2D\%2D-11-march-2020. Accessed 6 Apr 2020

2. Protezione civile. https://www.regione.lombardia.it/wps/portal/ istituzionale/HP/servizi-e-informazioni/enti-e-operatori/ protezione-civile. Accessed 3 Jun 2020

3. Mao L, Wang M, Chen S et al (2020) Neurological manifestations of hospitalized patients with COVID-19 in Wuhan, China: a retrospective case series study. medRxiv. https://doi.org/10.1101/2020. 02.22.20026500

4. Mao L, Jin H, Wang M et al (2020) Neurologic manifestations of hospitalized patients with coronavirus disease 2019 in Wuhan, China. JAMA Neurol. https://doi.org/10.1001/jamaneurol.2020. 1127

5. Politi LS, Balzarini L (2020) The radiology department during the COVID-19 pandemic: a challenging, radical change. Eur Radiol. https://doi.org/10.1007/s00330-020-06871-0

6. Zaidat OO, Lazzaro MA, Liebeskind DS, Janjua N, Wechsler L, Nogueira RG, Edgell RC, Kalia JS, Badruddin A, English J, Yavagal D, Kirmani JF, Alexandrov AV, Khatri P (2012) Revascularization grading in endovascular acute ischemic stroke therapy. Neurology 79:S110-S116. https://doi.org/10.1212/WNL. 0b013e3182695916

7. Ierardi AM, Wood BJ, Gaudino C et al (2020) How to handle a COVID-19 patient in the angiographic suite. Cardiovasc Intervent Radiol 43:820-826. https://doi.org/10.1007/s00270-020-02476-8
8. Pop R, Hasiu A, Bolognini F et al (2020) Stroke thrombectomy in patients with COVID-19: initial experience in 13 cases. AJNR Am J Neuroradiol. https://doi.org/10.3174/ajnr.A6750

9. Hernández-Fernández F, Sandoval Valencia H, Barbella-Aponte RA et al (2020) Cerebrovascular disease in patients with COVID19: neuroimaging, histological and clinical description. Brain 143: 3089-3103. https://doi.org/10.1093/brain/awaa239

10. Rothstein A, Oldridge O, Schwennesen H et al (2020) Acute cerebrovascular events in hospitalized COVID-19 patients. Stroke 51: e219-e222. https://doi.org/10.1161/STROKEAHA.120.030995

11. Rudilosso S, Laredo C, Vera V et al (2020) Acute stroke care is at risk in the era of COVID-19: experience at a comprehensive stroke center in Barcelona. Stroke 51:1991-1995. https://doi.org/10.1161/ STROKEAHA.120.030329

12. Nguyen TN, Abdalkader M, Jovin TG et al (2020) Mechanical thrombectomy in the era of the COVID-19 pandemic: emergency preparedness for neuroscience teams: a guidance statement from the society of vascular and interventional neurology. Stroke 51:18961901. https://doi.org/10.1161/STROKEAHA. 120.030100

13. Smith MS, Bonomo J, Knight WA et al (2020) Endovascular therapy for patients with acute ischemic stroke during the COVID-19 pandemic: a proposed algorithm. Stroke 51:1902-1909. https://doi. org/10.1161/STROKEAHA.120.029863

14. Li Y, Wang M, Zhou Y et al (2020) Acute cerebrovascular disease following COVID-19: a single center, retrospective, observational study. SSRN J. https://doi.org/10.2139/ssrn.3550025

15. Oxley TJ, Mocco J, Majidi S et al (2020) Large-Vessel Stroke as a presenting feature of Covid-19 in the young. N Engl J Med 382: e60. https://doi.org/10.1056/NEJMc2009787

Publisher's note Springer Nature remains neutral with regard to jurisdictional claims in published maps and institutional affiliations. 